\title{
Variations in gut bacterial communities of Hooded crane (Grus monacha) over spatial-temporal scales
}

\author{
Yuanqiu Dong ${ }^{1,2}$, Xingjia Xiang ${ }^{1,2}$, Guanghong Zhao ${ }^{1,2}$, Yunwei Song ${ }^{1,2,3}$, Lizhi Zhou ${ }^{\text {Corresp. } 1,2}$ \\ ${ }^{1}$ School of Resources and Environmental Engineering, Anhui University, Hefei, China \\ 2 Anhui Key Laboratory of Wetland Ecosystem Protection and Restoration, Anhui University, Hefei, China \\ 3 Anhui Province Shengjin Lake National Nature Reserve, Chizhou, China \\ Corresponding Author: Lizhi Zhou \\ Email address: zhoulz@ahu.edu.cn
}

Background: Microbes have been recognized as important symbionts to regulate host life. The animal gut harbors abundance and diverse bacteria. Numerous internal and external factors influence intestinal bacterial communities, including diet, seasonal fluctuations and habitat sites. However, the factors that influence the gut bacterial communities of wild bird is poorly characterized. Methods: By high-throughput sequencing and statistical analysis, we investigated the variations in gut bacterial communities of the hooded cranes at three wintering stages in Caizi (CZL) and Shengjin Lake (SJL), which are two shallow lakes in the middle and lower Yangtze River floodplain. Results: Our results revealed significant differences in gut bacterial community structure and diversity among different sampling sites and wintering stages. Seasonal changes have a significant impact on the gut microbe composition of Hooded cranes in the two lakes. ANOSIM analysis demonstrated that the samples in CZL had greater differences in the gut bacterial composition than that in SJL. Our data showed strong evidence that host's gut filtering might be an important factor in shaping bacterial community according to mean nearest taxon distance (MNTD). The PICRUSt analysis showed that the predicted metagenomes associated with the gut microbiome were carbohydrate metabolism, amino acid metabolism and energy metabolism over the entire wintering period at the two lakes. Conclusions: The results demonstrated that both seasonal changes and habitat sites have significant impact on the gut bacterial communities of Hooded cranes. In addition, predictive function of gut microbes in Hooded cranes varied over time. These results provide new insights into the gut microbial community of the cranes, which serves as a foundation for future studies. 
1 Title: Variations in gut bacterial communities of Hooded crane (Grus monacha) over spatial-

2 temporal scales

3 Authors: Yuanqiu Dong ${ }^{1,2}$, Xingjia Xiang ${ }^{1,2}$, Guanghong Zhao ${ }^{1,2}$, Yunwei Song ${ }^{1,2,3}$, Lizhi 4 Zhou $^{1,2^{*}}$

\section{Author Affiliation:}

6 1. School of Resources and Environmental Engineering, Anhui University, 111 Jiulong Road, 7 Hefei 230601, China

8 2. Anhui Province Key Laboratory of Wetland Ecosystem Protection and Restoration, Hefei 9 230601, Anhui University, China

10 3. Anhui Shengjin Lake National Nature Reserve, Chizhou 247200, China.

11

$12 *$ Corresponding author: Lizhi Zhou

13 Corresponding address: School of Resources and Environmental Engineering, Anhui 14 University, Hefei 230601, China.

15 Tel: +86-551-63861752, Fax: +86-551-63861752, E-mail: zhoulz@ahu.edu.cn 
16 Abstract:

17 Background: Microbes have been recognized as important symbionts to regulate host life. The animal gut harbors abundance and diverse bacteria. Numerous internal and external factors influence intestinal bacterial communities, including diet, seasonal fluctuations and habitat sites.

20 However, the factors that influence the gut bacterial communities of wild bird are poorly 21 characterized.

22 Methods: By high-throughput sequencing and statistical analysis, we investigated the variations 23 in gut bacterial communities of the Hooded cranes at three wintering stages in Caizi (CZL) and 24 Shengjin Lake (SJL), which are two shallow lakes in the middle and lower Yangtze River 25 floodplain.

26 Results: Our results revealed significant differences in gut bacterial community structure and 27 diversity among different sampling sites and wintering stages. Seasonal changes have a 28 significant impact on the gut microbe composition of Hooded cranes in the two lakes. ANOSIM 29 analysis demonstrated that the samples in CZL had greater differences in the gut bacterial composition than that in SJL. Our data showed strong evidence that host's gut filtering might be an important factor in shaping bacterial community according to mean nearest taxon distance (MNTD). The PICRUSt analysis showed that the predicted metagenomes associated with the gut microbiome were carbohydrate metabolism, amino acid metabolism and energy metabolism over 34 the entire wintering period at the two lakes.

36 Conclusions: The results demonstrated that both seasonal changes and habitat sites have 37 significant impact on the gut bacterial communities of Hooded cranes. In addition, predictive function of gut microbes in Hooded cranes varied over time. These results provide new insights into the gut microbial community of the cranes, which serves as a foundation for future studies.

41 Running title: Gut bacterial communities 


\section{Introduction:}

43 The gut microbiota confers mutualistic functions involved in substance synthesis and metabolism

44 (Eberl \& Boneca 2010), resistance to the intrusion of the pathogen (Guarner \& Malagelada 2003;

45 Koch \& Schmid-Hempel 2011) and modulation of immune development (Eberl \& Boneca 2010).

46 In vertebrates, the gut harbors diverse and abundant microbes that interact with host and

47 environmental factors to form a complex ecosystem (Qin et al. 2010). Previous studies showed

48 that gut microbiota are shaped by diet, life style (Nicholson et al. 2012) and environment factors

49 (e.g., seasonal fluctuations and location) (Hird et al., 2014).

50 Birds have unique life history traits that are different from other vertebrates, such as 51 migratory behavior, complex dietary habits, physiological traits and complex network of habitats, 52 all of which may impact gut microbial structure and function (Kohl 2012). Like other vertebrates, 53 the avian gut is colonized with abundant microbes. Previous studies of the intestinal microbiota 54 of birds have mainly focused on ornamental and economical birds, such as parrot (Waite et al. 55 2012), penguin (Dewar et al. 2013) and turkey (Wilkinson et al. 2017). However, little 56 information has been reported in wild birds. The rapid development in molecular methodologies 57 has provided new insight for the gut microbiota of birds. Recent research demonstrated that the 58 dominant phyla of the avian gut microbiota were Firmicutes, Proteobacteria, Actinobacteria and 59 Bacteroidetes (Waite and Taylor, 2014). Many studies have focused on captive birds which showed that the avian gut microbiota are affected by diet (Yang et al. 2016) and gut microbiota exhibited temporal stability (Kreisinger et al. 2017). Captivity also has been considered to be an important factor affecting the structure of avian gut microbes. Wild birds remained less studied despite for the association with pathogen transmission. Using the 16s rRNA high through sequencing, we tried to understand the diversity and potential functions of the gut microbiota in Hooded cranes and the effects of environmental factors on bird gut microbial communities.

Hooded cranes (Grus monacha) are large long-distance migratory colonial wading birds.

67 They are defined as a vulnerable species in the IUCN Red List of Threatened Species (Birdlife 68 2012) and Category I key National Protected Wild Animal Species in China. Hooded cranes 69 breed in south-central and south-eastern Siberia and Russia, and winter in Japan, China and 70 South Korea (Hammerson \& Ryan 2004). In China, the cranes still inhabit natural lakes and the 71 nearby paddy fields (Zheng et al., 2015). The food resources change over spatial-temporal scales. 72 The cranes had to modify their foraging patterns when food recourses change during the 
73 wintering periods (Wan et al. 2016; Zheng et al. 2015). A reduction in the food availability 74 forces animals to modify their foraging patterns (Zheng et al. 2015). In the early and mid-winter 75 periods, the most abundant, accessible and frequented food resources were found in paddy fields, 76 while in the late period the more abundant food were available in meadows. Hence, Hooded 77 cranes have changed their dietary structure and habitat. In China, Hooded cranes are omnivorous 78 birds, but feed mainly on plants (Huang \& Guo 2015; Zhao et al. 2002).

79 Shengjin and Caizi Lakes, the two-shallow river-connected lakes in the middle and lower 80 Yangtze River floodplain, respectively, are the most important wintering ground for cranes. 81 These lakes provide the birds with suitable feeding habitats during winter seasons (Chen et al. 82 2011). As a result of the lake degradation in the last decade, the cranes lost many suitable 83 foraging habitats. In this study, we used high-throughput sequencing methods to analyze the gut 84 bacterial community of Hooded cranes at three wintering stages in the two lakes. Based on spatial-temporal scales, we tested two hypotheses: (1) Gut bacterial diversity and composition, as measured by alpha- and beta-diversity, differs significantly in different wintering sites; (2) Gut bacterial composition exhibit the same pattern in different wintering location.

\section{Materials \& Methods}

90

\section{Ethics statement}

Fecal samples were collected after foraging to ensure that the Hooded cranes were devoid of human disturbance. It does not involve direct hunting or capture. Permission for the collection of fecal samples was obtained from the local government for wildlife management.

\section{Study areas}

The study was carried out in Shengjin $\left(30.25-30.50^{\circ} \mathrm{N}, 116.92-117.25^{\circ} \mathrm{E}\right)$ and Caizi $(30.75-$ $\left.30.97^{\circ} \mathrm{N}, 117.00-117.15^{\circ} \mathrm{E}\right)$ Lakes, which were located in the middle and lower Yangtze River floodplain (Figure 1). Both lakes are globally important wintering and stopover habitats for migratory wading birds on the East Asian-Australasian Flyway (Cao \& Fox 2009; Fox et al. 2011b). Shengjin and Caizi Lakes are river-connected shallow lakes with a northern subtropical monsoon climate. The average annual temperature is $14-18^{\circ} \mathrm{C}$ and the average annual rainfall is approximately $1000-1400 \mathrm{~mm}$. 
The seasonally inundated wetlands provide plenty of food (e.g., Oryza sativa, Polygonum

104

105

106

107

108

109

110

111

112

113

114

\section{Sample collection and preservation}

116 Fecal samples were collected during the three-wintering periods. The foraging sites of the crane 117 flocks were observed with a telescope before sampling to ensure that there were no other species.

118 To avoid human disturbance, the fecal samples were collected immediately upon the completion 119

120

121

122

123

124

125

126

127

128

129

130

131

132

133

criopolitanu, and Potentilla supina) for the wading birds (Zhao et al. 2013; Zheng et al. 2015).

There are approximately 600 individuals of Hooded cranes wintering in the two lakes each year.

The habitat utilization rate of the cranes has shifting over spatial and time scales (Zhao et al. 2013; Zheng et al. 2015). During the wintering period, shifts in food density and resources triggers cranes to adjust their foraging habitats (Wan et al. 2016; Zhao et al. 2013; Zheng et al. 2015). The wintering period can be divided into three stages according to the migratory rhythm and hydrological processes of the cranes: the early stage from November to December; the middle stage from January to February; and the late stage from March to April (Zhao et al. 2013; Zheng et al. 2015). The foraging habitats of Hooded cranes are relatively stable at certain wintering stage in Shengjin and Caizi Lakes (Cao et al. 2010).

of foraging. Fecal samples were collected at a minimum distance of approximately $1-2 \mathrm{~m}$ to avoid individual sampling repetition (Zhang \& Zhou 2012). All samples were obtained from inside the feces to avoid soil contaminants. The samples were kept in a cooler, transported under refrigeration to the lab within several hours and stored at $-80^{\circ} \mathrm{C}$. A total of 87 fecal samples were used in this study. 43 samples were collected from Shengjin Lake (SJL) and 44 samples from Caizi Lake (CZL). In Shengjin Lake, the samples were 13 in the early wintering period (SJL-E), 14 in the middle wintering period (SJL-M) and 15 in the late wintering period (SJL-L). In Caizi Lake, the samples were 14 in the early wintering period (CZL-E), 15 in the middle wintering period (CZL-M), and 15 in the late wintering period (CZL-L).

\section{DNA extraction and species determination}

DNA was extracted from the fecal samples using the Qiagen QIAamp ${ }^{\circledR}$ DNA Stool Mini Kit following the DNA isolation protocol for pathogens. DNA was eluted in $150 \mu \mathrm{L}$ of sterilized 
134 deionized water and then stored at $-80{ }^{\circ} \mathrm{C}$. The COI gene was amplified using the BIRDF1135 BIRDR1 primer pair to determine host species. PCR amplification was performed in a $50 \mu \mathrm{L}$ 136 reaction volume containing $100 \mathrm{ng}$ of fecal DNA, $25 \mu \mathrm{L}$ of $2 \times$ EasyTaq mix (TransGen), $10 \mu \mathrm{M}$ 137 BIRDF1 (TTCTCCAACCACAAAGACATTGGCAC) and $10 \mu \mu$ BIRDR1 138 (ACGTGGGAGATAATTCCAAATCCTG). The cycling conditions were as follows: $95^{\circ} \mathrm{C}$ for 5 $139 \mathrm{~min}$, followed by 30 cycles of $95{ }^{\circ} \mathrm{C}$ for $30 \mathrm{~s}, 55{ }^{\circ} \mathrm{C}$ for $45 \mathrm{~s}$ and $72{ }^{\circ} \mathrm{C}$ for $90 \mathrm{~s}$, with a final 140 extension period at $72{ }^{\circ} \mathrm{C}$ for $10 \mathrm{~min}$. The PCR products were sequenced by Sangon Biotech Co. 141 Ltd. (Shanghai). The sequences were aligned by NCBI. All of the samples were confirmed to 142 contain Hooded cranes DNA based on the sequence analysis.

143

144 16S rRNA sequencing

145 Primer sets 338F/806R equipped with sequencing adapters and unique identifier tags were used 146 to amplify the V3-V4 hypervariable regions of the bacterial 16S rRNA genes fragments for the 147 Illumina Hiseq platform (PE250). PCR was carried out in $50 \mu \mathrm{L}$ volume containing $60 \mathrm{ng}$ of 148 fecal DNA, $25 \mu \mathrm{L}$ of $2 \times$ Premix Taq and $1 \mu \mathrm{L}$ each of the forward and reverse primers $(10 \mu \mathrm{M})$.

149 The cycling conditions were as follows: $94^{\circ} \mathrm{C}$ for $3 \mathrm{~min}$, followed by 30 cycles of $95^{\circ} \mathrm{C}$ for $30 \mathrm{~s}$, $15055^{\circ} \mathrm{C}$ for $45 \mathrm{~s}$ and $72{ }^{\circ} \mathrm{C}$ for $45 \mathrm{~s}$, with a final extension at $72{ }^{\circ} \mathrm{C}$ for $10 \mathrm{~min}$. Triplicate reaction 151 mixtures per sample were pooled together and purified using the EZNA Gel Extraction Kit 152 (Omega, USA). Quantification was performed with a NanoDrop. Sequences were processed 153 using Quantitative Insights Into Microbial Ecology (QIIME). After denoising, poor quality 154 (below an average quality score of 30) and short sequences (shorter than 200bp) were removed 155 and those sequences which exactly matched with their barcode and primer were kept. The 156 barcode and primer sequences were then removed. Sequences were clustered into OTUs using a $15797 \%$ identity threshold by UPARSE. Singleton OTUs were deleted and chimers were filtered to 158 remove erroneous OTUs due to sequence errors using the USEARCH in QIIME. The most 159 abundant sequence within each cluster was selected as the representative sequence for that OTU. 160 The taxonomic identify of each OTU was checked against the ribosomal database project (RDP) 161 Classifier. The raw data has been submitted to SRA of NCBI with accession number 162 SUB2178492.

163

164 Data analysis 
165 Non-metric multidimensional scaling (NMDS) based on the Bray-Curtis dissimilarity (calculated 166 from the relative abundance matrix) and Analyses of Similarities (ANOSIM; permutations = 999) 167 were performed to compare the community composition in different treatments in R 3.4.1 (vegan 168 2.4-3) (Dixon 2010). In addition, permutational analysis of multivariate dispersions (PERMDISP; 169 permutations $=999$ ) was used to test the gut bacterial community structure heterogeneity varied 170 over spatial-temporal scale. This test was using a Bray-Custis similarity matrix. The significance 171 of PERMDISP was determined via the ANOVA F-statistic to compare among-group differences 172 in the distance from observations to their corresponding group centroids. One-way and two-way 173 ANOVA was used to test observed species, PD whole tree, Shannon and Chao 1 indices among 174 different treatments. The nearest taxon index (NTI) and betaNTI are used to test the assembly 175 processes of the gut bacterial community. NTI was calculated to assess the spatial and temporal 176 changes in bacterial phylogenetic structure using Picante software. The NTI can be used to test 177 for phylogenetic clustering or overdispersion. Positive NTI values and low quantiles $(P<0.05)$ 178 indicate that co-occurring species are more closely related than expected by chance (clustering), 179 whereas positive values and high quantiles $(P>0.05)$ indicate that the co-occurring species are 180 less closely related than expected by chance (overdispersion). Positive betaNTI values indicate 181 greater than expected phylogenetic turnover, and betaNTI was calculated in phylocom. LEfSe 182 (linear discriminant analysis effect size) was used to identifies genomic features characterizing 183 the differences between two or more biological conditions (Segata et al. 2011). To detect KEGG 184 pathways with significantly different abundances between the two lakes, LEfSe analysis was 185 used according to the online protocol (https:// huttenhower. sph. harvard. edu/galaxy/). Nearest 186 sequenced taxon index (NSTI) was the sum of phylogenetic distances for each organism in the 187 OTU table and the closest genetic relationship of the sequencing reference genome, as measured 188 by the substitutions per site in the $16 \mathrm{~S}$ rRNA gene and weighted by the frequency of that 189 organism in the OTU table (Langille et al. 2013). Functional predictions were made based on the 16S rRNA OTU membership using PICRUSt (Phylogenetic Investigation of Communities by 191 Reconstruction of Unobserved States) according to the online protocol 192 (http://picrust.github.io/picrust/). One-way ANOVA was used to detect the influence of the 193 sampling site and season on bacterial taxonomy (phylum) variation. All univariate statistical 194 analyses were conducted using SPSS 20.0. 
196 Results

\section{Bacterial alpha-diversity}

198 Gut bacterial alpha-diversity was calculated at a depth of 10,000 randomly selected sequences 199 per sample. Alpha-diversity was estimated by the observed species index, phylogenetic diversity, 200 Shannon and Chao 1 index. Two-way ANOVA showed that all alpha diversity was significantly 201 different with time, and only phylogenetic diversity and Chao 1 index were significantly 202 different in spatial variation. Alpha diversity was significantly different under the interaction of 203 wintering period and spatial distance (Table S1). At the early wintering periods, the alpha204 diversity of the SJL samples were significantly higher than that of the CZL samples $(P<0.001)$

205 (Figure 2), but there was no significant difference in middle and late wintering periods between 206 the two lakes $(P>0.05$ in both causes). Alpha-diversity was the highest in the middle period 207 across the temporal changes in CZL, and the Chao 1 index of the SJL samples showed 208 significant different across the temporal change (Figure S1).

209

210 Bacterial community composition

211 In total, we obtained 4,717,488 reads of high-quality sequences with an average of 46,193 reads 212 was found. Potentially chimeric sequences and singleton OTU $(698,694)$ were then discarded. 213 The dominant gut bacterial phyla were Firmicutes $(59.82 \% \pm 32.35 \%)$, Proteobacteria $(26.82 \% \pm$ $21424.87 \%)$, Actinobacteria $(5.43 \% \pm 8.19 \%)$, Fusobacteria $(3.78 \% \pm 12.22 \%)$, and Bacteroidetes $215(2.24 \% \pm 6.21 \%)$ (Figure 3A, C). Within Firmicutes, the dominant bacterial classes were 216 Clostridia $(41.79 \pm 31.94 \%)$ and Bacilli $(17.96 \pm 21.17 \%)$. The classes of Epsilonproteobacteria 217 (12.61 $\pm 21.80 \%)$, Gamaproteobacteria (8.69 $\pm 13.87 \%)$ and Alphaproteobacteria $(4.28 \pm 8.04 \%)$ 218 were dominant in Proteobacteria (Figure 3B, D). However, the distribution of each taxon among 219 the four groups was uneven, as indicated by Figure 3. At the family level, the top 30 families 220 were shown for all samples (Figure S2). At the lower level, only $84.7 \%$ of sequences could be 221 assigned to 995 genera. The dominate genera were Clostridium (15.5\%), SMB53 (13.62\%), 222 Helicobacter (11.86\%), Lactobacillus (7.22\%), Epulopiscium (4.82\%), Enterococcus (4.76\%), 223 Fusobacterium (3.67\%), Pseudomonas (2.54\%), Turicibacter (2.08\%), Serratia (2.03\%), 224 Agrobacterium (1.77\%) and Lysinibacillus (1.59\%).

225 One-way ANOVA showed that relative abundance of Firmicutes was significantly higher in 226 SJL samples than in CZL samples, whereas the relative abundance of Proteobacteria from the 
227 SJL samples was significantly lower than the CZL samples (Figure 4, S3). At Shengjin Lake, the 228 relative abundance of the Firmicutes increased significantly along the winter period. In order to 229 explore the differences in spatial and temporal scale, we conducted LEfSe tests to detect the 230 difference in relative abundance of microbial taxa. At Shengjin Lake, four indicator bacterial 231 taxa (i.e., Pseudomonadaceae, Pseudomonas, Sphingobacteriales, Sphingobacterii) were found 232 in the gut of the Hooded crane in SJL-E samples and five indicator bacterial taxa (i.e., 233 Clostridiales, Clostridia, Lachnospiraceae, Epulopiscium, Peptostreptococcaceae) were found 234 in the gut of Hooded crane in SJL-L samples (Figure 5A, B). At Caizi Lake, three indicator 235 bacterial taxa (Fusobacteriaceae, Fusobacterium, Peptostreptococcaceae) were found in the 236 CZL-E samples, four indicator bacterial taxa (i.e., Sphingobacteriaceae, Sphingobacterii, 237 Sphingobacteriales, Microccaceae) were found in the CZL-M samples and 11 indicator bacterial 238 taxa (Gammaproteobacteria, Enterobacteriaceae, Enterobacteriales, Bacilli, Lactobacillales, 239 Enterococcaceae, Enterococcus, Lactobacillales, Enterococcus, Lactobacillus, Lactobacillaceae, 240 Enterobacter) were found in the CZL-L samples (Figure 5C, D).

241 The bacterial community compositions were significantly different between the guts of 242 Hooded crane from SJL samples and CZL samples. NMDS revealed that SJL samples tended to 243 be less different compared to the CZL samples (Figure 6E). ANOSIM analysis confirmed that 244 seasonal changes had a significant impact on the microbial composition of the Hooded cranes in 245 two lakes (Table 1). The bacterial community composition of SJL samples and CZL samples 246 were significantly different $(P=0.001)$ in the three wintering periods (Table 1$)$, the bacterial

247 community compositions in different wintering period were significantly different in both 248 Shengjin and Caizi Lake $(\mathrm{R}=0.238, P=0.001)$ (Table 1$)$.

According to PERMDISP, average distance to the corresponding group centroid have no significant differences among different wintering periods at Shengjin Lake $(P=0.167)$. However, mean distance to the corresponding group centroid have significant differences among different wintering periods at Caizi Lake $(P<0.001)$. Furthermore, no significant differences could be

253 detected among average distance to the corresponding group centroid of gut bacterial 254 communities in different sample collected sites.

255

Assemblage processes of the gut bacterial community 
257 NTI was used to evaluate the gut bacterial phylogenetic structure. All of the NTI values were 258 positive, which showed that the bacterial communities were phylogenetically clustered (Figure 7, 259 S4). At the early wintering stage, the CZL-E samples NTI values were less positive compared to 260 the SJL-E samples, which indicated that phylogenetic clustering was weakest in the CZL-E 261 samples (Figure 7). In addition, the NTI values of SJL-L samples were less positive compared to 262 the CZL-L samples at late wintering stage, which indicated that phylogenetic clustering was 263 weakest in the SJL-L samples. Phylogenetic clustering was similar in SJL samples during three 264 wintering periods, whereas CZL-L samples phylogenetic clustering were more similar in CZL 265 samples.

266

\section{Variation in predicted metagenomes between the two lakes}

268 In addition, NSTI also influenced PICRUSt accuracy. The NSTI for our samples was $0.18 \pm 0.07$

269 similar to the previously reported analyses in soils (mean NSTI $=0.17 \pm 0.02$ ). In this study, a 270 total of 41 functional genes were predicted in Hooded crane population. The majority of 271 functions were membrane transport (12.63\%), carbohydrate metabolism $(9.36 \%)$, amino acid 272 metabolism (9.19\%), replication and repair (8.08\%), energy metabolism (7.10\%) and translation $273(5.18 \%)$ during the wintering period. ANOSIM analysis revealed that the potential functions of 274 the gut bacterial communities of the two lakes were significantly different during early and late 275 wintering periods $(P=0.001)$ (Table 2). However, during the middle wintering period, there was 276 little difference in the potential functions of the gut bacterial in the two lakes $(P=0.116)$. In 277 early wintering period, Hooded crane from Caizi Lake were predicted to have a microbiota with 278 enhanced capability for metabolic disease, carbohydrate metabolism, amino acid metabolism, 279 circulatory transport, cellular processes and singling and a lower rate of microbial genes 280 associated with genetic information processing (Table S2). In middle wintering period, Hooded 281 crane from Caizi Lake were predicted to have a microbiota with enhanced capability for amino 282 acid metabolism, sensory system, transport and catabolism, membrane transport and genetic 283 information processing. When comparing predicted metagenomes in the late period, the 284 microbiota of Hooded crane from Caizi Lake was predicted to have a capability for metabolic 285 disease, carbohydrate metabolism, amino acid metabolism, replication and repair and glycan 286 biosynthesis and metabolism. 


\section{Discussion}

288 Hooded cranes have to modify their foraging behavior to adapt to variations in food availability 289 over a spatial-temporal scale as a result of wetland loss and degradation (Wan et al. 2016; Zheng 290 et al. 2015). Having a diverse gut microbiome may be another adaptive mechanism. In this study, 291 the gut bacterial composition and structure of the wintering Hooded Crane from the Shengjin and 292 Caizi Lakes were explored. Differences in microbial community structures and interactions were 293 also identified.

294 The gut microbial community of the Hooded crane was dominated by Proteobacteria, 295 Firmicutes and Actinobacteria, which was similar to that of mammals (Waite \& Taylor 2014).

296 The phylum of Firmicutes was dominated in Hooded crane gut at the two lakes and was 297 consistent with the many avian species (Dewar et al. 2014; Waite \& Taylor 2014; Wilkinson et al. 298 2017). Firmicutes are associated with the breakdown of carbohydrates, polysaccharides, sugars 299 and fatty acids, which are utilized by the host as energy sources (Flint et al. 2008). The 300 clostridium genus belonging to Firmicutes can digest simple carbohydrates (Aristilde 2017; 301 Bäckhed et al. 2004) as well as complex polysaccharides (Aristilde 2017; Ramos et al. 2015), 302 which may lead to high proportion of carbohydrates metabolism. All above results indicated that 303 the Hooded cranes, like other waterbirds, feed on high-energy foods such as Vallisneria tubers 304 (Fox et al. 2011a; Li-Lin et al. 2008). In order to survive in the dry and cold weather, the Hooded 305 cranes need considerable amounts of energy (Cai et al. 2014; Fox et al. 2011a). However, 306 Proteobacteria plays an important role in energy accumulation (Bryant \& Small 1956; Chevalier 307 et al. 2015), and the high proportion of Proteobacteria may be due to the cranes need a lot of 308 energy to cope with the cold winter. The classes of Epsilonproteobacteria, Gamaproteobacteria 309 and Alphaproteobacteria were dominant in Proteobacteria, similar to other avian, such as 310 kittiwake, bean goose, kakapo (Dongen et al. 2013; Yang et al. 2016). The dominant families 311 were Xanthomonadaceae and Comamonadaceae. These two families are strong competitors with

312 flexible metabolism and are considered essential for host's digestion in poor nutritional diet. The

313 phylum Fusobacteria associated with butyrate production, with butyrate playing an important 314 role in ion absorption and immune regulation. It is also an important anti-inflammatory agent 315 (Canani et al. 2011). The phylum of Actinobacteria also found in the Hooded cranes and 316 associated with pathogens, such as the genera of Nocardia and Rhodococcus (Santos et al. 2012).

317 The habitats of domestic waterbirds overlapping with Hooded cranes at the two lakes, and large 
318 assemblance of migratory birds may represent a source of pathogenic microbes that can be 319 transmitted by each other through feces (Zhao et al., 2017).

320 However, the detailed composition of these phyla was notably altered with space-time 321 according to our study. The samples from Shengjin Lake were dominated by Firmicutes, while 322 those from Caizi Lake were dominated by Firmicutes and Proteobacteria. Compared to Shengin 323 Lake, the samples from the Caizi Lake appear to be enriched in Proteobacteria and the relative 324 abundance of Firmicutes decreased (Figure S1). Increased the relative abundance of 325 Proteobacteria in the gut microbiota increase the risk of inflammatory bowel disease (Zhou et al. 326 2016). According to LEfse analysis, genus of Pseudomonas was enriched in the gut of Hooded 327 crane from SJL-E samples. Pervious study shows that the Pseudomonas genus codes for 328 levansucrases (Visnapuu et al. 2015). We also found the class of sphingobacteriales were highest 329 in the gut of Hooded crane from SJL-E samples and CZL-M samples (Figure 5) because of the 330 increase in polysaccharide (Carney et al. 2014). In addition, there was no significant difference 331 in the alpha diversity of the SJL samples in different wintering periods, while the alpha diversity 332 of the CZL was significantly difference in samples in different wintering periods (Figure S1). 333 Furthermore, significant differences in gut microbial communities were identified, as reflected 334 by NMDS clustering and microbial interactions (Figure 4, Table 1). These results showed that 335 the seasonal and temporal change may be important factors in shaping host bacterial structure. In 336 recent years, grazing animal waste and poultry litter effects on the environment (Sauer et al. 337 1999). As most members of genera (i.e. Agrobacterium) are present in soil, these bacteria may 338 have originated from the environment, implying horizontal transmission could also influence the 339 structure of animal gut microbiota (Yang et al. 2016). Thus, grazing animal waste and poultry 340 litter also affects the structure of the gut microbiota. Seasonal fluctuations also had a significant 341 effect on the composition of gut microbes at the two lakes.

342 In addition, all the bacterial community assemblages showed significant phylogenic 343 clustering, indicating that bacterial communities were strongly structured by gut environment 344 filtering (Yan et al. 2016). Although the bacterial community compositions showed significantly 345 different between the two sites in the early wintering period, however, the spatial changes did not 346 affect bacterial phylogenetic clustering, while gut environmental filtering influenced bacterial 347 communities in both lakes in the middle and late periods. The effect of temporal changes on 348 bacterial phylogenetic structure was not consistent in Shengjin and Caizi lakes. These results 
349 suggest that the environment factors can influence the composition of bacterial community and 350 might be an important factor for bacterial phylogenetic structure.

351 The bacterial community in the guts of Hooded cranes may have many important functions.

352 In this study, PICRUSt were used to deduce potential gene profiles from 16S rRNA sequencing.

353 The result showed that the most abundant functional classes were related to membrane transport, 354 carbohydrate, amino acid and energy metabolism. The higher proportion of energy metabolism 355 pathway-related predicted genes may meet the Hooded crane's energy needs for flight. The 356 energy metabolism pathways were much higher in the late wintering than in the other two 357 periods, which may be induced by the fact that the food density gradually decreases over winter 358 period, leading to an increase in the foraging efforts of Hooded cranes (Wan et al. 2016). 359 Moreover, the PICRUSt results revealed that the glycan biosynthesis and metabolism-related 360 genes were present in both lakes but with higher proportion at Caizi Lake. Therefore, we propose that this pathway in the gut microbiota may significantly contribute to increase digestive

362

363 efficiency and assimilation, which may play an important role in providing energy and nutrients to cope with the cold weather (Wan et al. 2016).

364

365

366

367

368

369

370

371

372

373

374

375

376

377 analysis.

378

379

\section{Conclusions}

In this study, we showed that gut bacterial community composition and diversity of Hooded cranes changed over spatial-temporal scales. We found that spatial-temporal changes might be important factors to influence gut bacterial community composition. In addition, dietary seasonal fluctuation also affected the gut bacterial community composition. This study provides a foundation understanding of gut bacterial community composition and potentially bacterial functions in Hooded cranes. Future work should focus on how these actual functions relate to the gut microbial community composition.

\section{Acknowledgments}

We appreciate the help from the staff of Shengjin Lake Nature Reserve Bureau and local villagers. We are grateful to Binghua Sun, Nazia Mehtab and Yang Li for their assistance in data

\section{References}

PeerJ reviewing PDF | (2018:11:32511:2:1:NEW 19 Apr 2019) 
380 381

382

383 384

385

386

387 388

389

390

391

392

393

394

395

396

397

398

399

400

401

402

403

404

405

406

407

408

409

410

Aristilde L. 2017. Metabolite labelling reveals hierarchies in Clostridium acetobutylicumthat selectively channel carbons from sugar mixtures towards biofuel precursors. Microbial Biotechnology 10:162-174.

Bäckhed F, Ding H, Wang T, Hooper LV, Koh GY, Nagy A, Semenkovich CF, and Gordon JI. 2004. The gut microbiota as an environmental factor that regulates fat storage. Proceedings of the National Academy of Sciences of the United States of America 101:15718.

Bryant MP, and Small N. 1956. Characteristics of two new genera of anaerobic curved rods isolated from the rumen of cattle. Journal of Bacteriology 72:22.

Cai T, Huettmann F, and Guo Y. 2014. Using stochastic gradient boosting to infer stopover habitat selection and distribution of Hooded Cranes Grus monacha during spring migration in Lindian, Northeast China. PLoS ONE 9:e89913.

Canani RB, Costanzo MD, Leone L, Pedata M, Meli R, and Calignano A. 2011. Potential beneficial effects of butyrate in intestinal and extraintestinal diseases. World Journal of Gastroenterology 17:1519-1528.

Cao L, and Fox AD. 2009. Birds and people both depend on China's wetlands. Nature 460.

Carney LT, Reinsch SS, Lane PD, Solberg OD, Jansen LS, Williams KP, Trent JD, and Lane TW. 2014. Microbiome analysis of a microalgal mass culture growing in municipal wastewater in a prototype OMEGA photobioreactor. Algal Research 4:52-61.

Chen JY, Zhou LZ, Zhou B, Xu RX, Zhu WZ, and Xu WB. 2011. Seasonal dynamics of wintering waterbirds in two shallow lakes along Yangtze River in Anhui Province. Zoological Research 32:540-548.

Chevalier C, Stojanović O, Colin DJ, Suarez-Zamorano N, Tarallo V, Veyrat-Durebex C, Rigo D, Fabbiano S, Stevanović A, and Hagemann S. 2015. Gut microbiota orchestrates energy homeostasis during cold. Cell 163:1360.

Dewar ML, Arnould JPY, Dann P, Trathan P, Groscolas R, and Smith S. 2013. Interspecific variations in the gastrointestinal microbiota in penguins. Microbiologyopen 2:195.

Dewar ML, Arnould JPY, Krause L, Dann P, and Smith SC. 2014. Interspecific variations in the faecal microbiota of Procellariiform seabirds. FEMS Microbiology Ecology 89:47.

Dixon P. 2010. VEGAN, a package of $\mathrm{R}$ functions for community ecology. Journal of Vegetation Science 14:927-930. 
411 Dongen V, Wouter FD, Brandl, Hanja B, Moodley, Yoshan, Wagner, Richard H, White, and Joel.

412

413

414

415

416

417

418

419

420

421

422

423

424

425

426

427

428

429

430

431

432

433

434

435

436

437

438

439

440
2013. Age-related differences in the cloacal microbiota of a wild bird species. $B M C$ Ecology 13:11-11.

Eberl G, and Boneca IG. 2010. Bacteria and MAMP-induced morphogenesis of the immune system. Current Opinion in Immunology 22:448-454.

Flint HJ, Bayer EA, Rincon MT, Lamed R, and White BA. 2008. Polysaccharide utilization by gut bacteria: potential for new insights from genomic analysis. Nature Reviews Microbiology 6:121-131.

Fox AD, Cao L, Zhang Y, Barter M, Zhao MJ, Meng FJ, and Wang SL. 2011a. Declines in the tuber-feeding waterbird guild at Shengjin Lake National Nature Reserve, China \&ndash; a barometer of submerged macrophyte collapse. Aquatic Conservation Marine \& Freshwater Ecosystems 21:82-91.

Guarner F, and Malagelada J. 2003. Gut flora in health and disease. Lancet 361:512.

Hammerson G, and Ryan TJ. 2004. The IUCN Red List of Threatened Species. IUCN Red List of Threatened Species.

Huang J, and Guo Y. 2015. Diet of Hooded Crane (Grus monacha)in Autumn,Lindian,China. Chinese Journal of Wildlife.

Koch H, and Schmid-Hempel P. 2011. Socially transmitted gut microbiota protect bumble bees against an intestinal parasite. Proceedings of the National Academy of Sciences of the United States of America 108:19288-19292.

Kohl KD. 2012. Diversity and function of the avian gut microbiota. Journal of Comparative Physiology B 182:591-602.

Kreisinger J, Kropáčková L, Petrželková A, Adámková M, Tomášek O, Martin JF, Michálková R, and Albrecht T. 2017. Temporal stability and the effect of transgenerational transfer on fecal microbiota structure in a long distance Migratory Bird. Frontiers in Microbiology 8:50.

Langille MGI, Zaneveld J, Caporaso JG, Mcdonald D, Dan K, Reyes JA, Clemente JC, Burkepile DE, Thurber RLV, and Knight R. 2013. Predictive functional profiling of microbial communities using $16 \mathrm{~S}$ rRNA marker gene sequences. Nature Biotechnology 31:814. 
441 Li-Lin XU, Wen-Bin XU, Sun QY, Zhou ZZ, Shen J, and Zhao XX. 2008. Flora and vegetation 442 in Shengjin Lake. Journal of Wuhan Botanical Research.

443 Nicholson JK, Holmes E, Kinross J, Burcelin R, Gibson G, Jia W, and Pettersson S. 2012. Host444 gut microbiota metabolic interactions. Science 336:1262-1267.

445 Qin J, Li R, Raes J, Arumugam M, Burgdorf KS, Manichanh C, Nielsen T, Pons N, Levenez F, 446 and Yamada T. 2010. A human gut microbial gene catalogue established by metagenomic 447 sequencing. Nature 464:59-65.

448 Ramos JL, Sol CM, Molina-Santiago C, Segura A, Duque E, Gómez-García MR, Udaondo Z, 449 and Roca A. 2015. Mechanisms of solvent resistance mediated by interplay of cellular 450 factors in Pseudomonas putida. FEMS Microbiology Reviews 39:555.

451

452

453

454

455

456

457

458

459

460

461

462

463

464

465

466

467

468

469

Santos SS, Pardal S, Proenã§A DN, Lopes RJ, Ramos JA, Mendes L, and Morais PV. 2012. Diversity of cloacal microbial community in migratory shorebirds that use the Tagus estuary as stopover habitat and their potential to harbor and disperse pathogenic microorganisms. FEMS Microbiology Ecology 82:63-74.

Sauer TJ, Daniel TC, Moore PA, Coffey KP, Nichols DJ, and West CP. 1999. Poultry Litter and Grazing Animal Waste Effects on Runoff Water Quality. Journal of Environmental Quality 28:860-865.

Segata N, Izard J, Waldron L, Gevers D, Miropolsky L, Garrett WS, and Huttenhower C. 2011. Metagenomic biomarker discovery and explanation. 12:R60.

Visnapuu T, Mardo K, and Alamäe T. 2015. Levansucrases of a Pseudomonas syringae pathovar as catalysts for the synthesis of potentially prebiotic oligo- and polysaccharides. New Biotechnology 32:597-605.

Waite DW, Deines P, and Taylor MW. 2012. Gut microbiome of the critically endangered New Zealand Parrot, the Kakapo (Strigops habroptilus). PLoS ONE 7:e35803.

Waite DW, and Taylor MW. 2014. Characterizing the avian gut microbiota: membership, driving influences, and potential function. Frontiers in Microbiology 5:223.

Wan W, Zhou L, and Song Y. 2016. Shifts inforaging behavior ofwintering Hooded Cranes (Grus monacha) inthree different habitats at Shengjin Lake, China. Avian Research 7:167-173. 
470 Wilkinson TJ, Cowan AA, Vallin HE, Onime LA, Oyama LB, Cameron SJ, Gonot C, Moorby

471

472

473 474

475

476

477

478

479

480

481

482

483

484

485

486

487

488

489

490

491
JM, Waddams K, and Theobald VJ. 2017. Characterization of the microbiome along the gastrointestinal tract of growing Turkeys. Frontiers in Microbiology 8:1089.

Yan Q, Li J, Yu Y, Wang J, He Z, Van Nostrand JD, Kempher ML, Wu L, Wang Y, and Liao L. 2016. Environmental filtering decreases with fish development for the assembly of gut microbiota. Environmental Microbiology 18:4739.

Yang Y, Deng Y, and Cao L. 2016. Characterising the interspecific variations and convergence of gut microbiota in Anseriformes herbivores at wintering areas. Scientific Reports $6: 32655$.

Zhang L, and Zhou L. 2012. Genetic structure of wintering Hooded Cranes (Grus monacha) based on mitochondrial DNA D-loop sequences.

Zhao F, Zhou L, Xu W, Zhao F, Zhou L, and Xu W. 2013. Habitat utilization and resource partitioning of wintering Hooded Cranes and three goose species at Shengjin Lake. Chinese Birds 4:281-290.

Zhao Y, Ma Z, and Chen J. 2002. Food habits of hooded crane (Grus monacha) in winter at the east tidelands of Chongming island. Journal of Fudan University.

Zheng M, Zhou L, Zhao N, and Xu W. 2015. Effects of variation in food resources on foraging habitat use by wintering Hooded Cranes (Grus monacha). Avian Research 6:1-10.

Zhou Y, He Y, Xu ZZ, Yang Y, Nie Y, Knight R, Zhou H, and Chen Y. 2016. Tu1963 Shift From Firmicutes-Enriched to Proteobacteria-Enriched and Specific Clostridials Reduction in Intestinal Microbiota Accociate With Activity of Inflammatory Bowel Disease. Gastroenterology 150:S992-S992. 
492 Figure legends

493 Figure1. The sampling areas of in this study.

494 Figure2. Variations in alpha-diversity ((A) Phylogenetic diversity, (B) OTU richness, (C) Chao

4951 and (D) Shannon) of different wintering periods. Different letters represent significant 496 differences by Tukey's HSD comparisons $(P<0.05)$. Error bars indicate standard deviation.

497 Figure3. Relative abundance of gut bacteria at the phylum, class and genus levels at two lakes.

498 (A) Relative abundance of the dominant phyla in all samples. (B) Relative abundance of the 499 dominant classes in all samples; Rings represent corresponding phylum (Firmicutes in blue and 500 Proteobacteria in red) for each of the most frequently represented class. (C) Relative abundance 501 at the phylum and (D) relative abundance at the genera at different periods in the two lakes.

502 Figure4. Relative abundance of the dominant phyla ( (A) Firmicutes, (B) Ptoteobacteria, (C) 503 Actinobacteria, (D) Fusobacteria) in different wintering periods. Samples are grouped according 504 to sampling location and wintering periods. Different letters represent significant differences by 505 Tukey's HSD comparisons $(P<0.05)$. Error bars indicate standard deviation.

506 Figure5. LEFSe analysis of the Hooded Crane guts bacteria in different wintering periods (LDA > $5072, P<0.05$ ). (A) LDA score representing the taxonomic with significant difference in different 508 wintering periods at the Shengjin Lake. (B) Cladogram representing the taxonomic hierarchical 509 structure of the phylotype biomakera identified among three wintering periods from the Shengjin 510 Lake; Red, phylotypes statistically overrepresented in early wintering period; Green, phylotypes 511 statistically overrepresented in late wintering period. (C) LDA score representing the taxonomic 512 with significant difference in different wintering periods at the Caizi Lake. (D) Cladogram 513 represting the taxonomic hierarchical structure of the phylotype biomakera identified among 514 three wintering periods from the Caizi Lake; Red, phylotypes statistically overrepresented in 515 early wintering period; Green, phylotypes statistically overrepresented in late wintering period; 516 Blue, phylotypes statistically overrepresented in middle wintering period.

517 Figure6. Differences in gut bacterial community composition. (A) Non-metric multidimensional 518 scaling plot bacterial community composition from Shengjin and Caizi Lakes at three wintering 519 periods. (B) Non-metric multidimensional scaling plot showing bacterial community 520 composition from Shengjin and Caizi Lakes at the early wintering period. (C) Non-metric

521 multidimensional scaling plot for the hooded crane from Shengjin and Caizi Lakes at the middle 522 wintering period. (D) Non-metric multidimensional scaling plot for the hooded crane from 
523 Shengjin and Caizi Lakes at the late wintering period. (E) Non-metric multidimensional scaling 524 plot for the hooded crane from Shengjin Lake at three wintering periods. (F) Non-metric 525 multidimensional scaling plot for the hooded crane from Caizi Lake at three wintering periods.

526 Figure7. Bacterial phylogenetic structure evaluated by the NTI in different wintering periods. (A) 527 At the early wintering period. (B) At the middle wintering period. (C) At the late wintering 528 period.

529 
Figure 1

Sampling areas

The sampling areas of in this study.

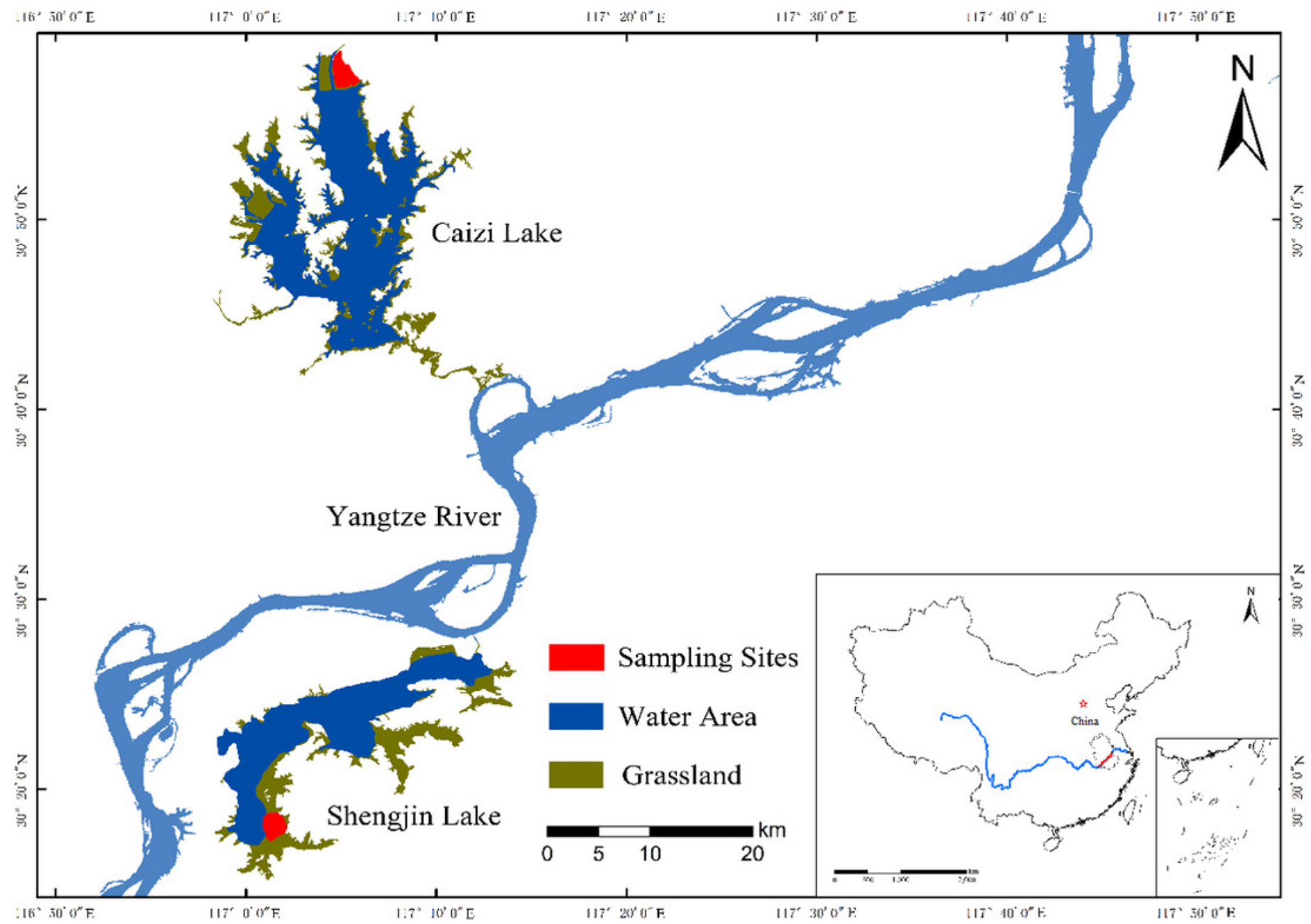


Figure 2

Alpha-diversity of different wintering periods.

Variations in alpha-diversity ((A) Phylogenetic diversity, (B) OTU richness, (C) Chao 1 and (D) Shannon) of different wintering periods. Different letters represent significant differences by Tukey's HSD comparisons $(P<0.05)$. Error bars indicate standard deviation.

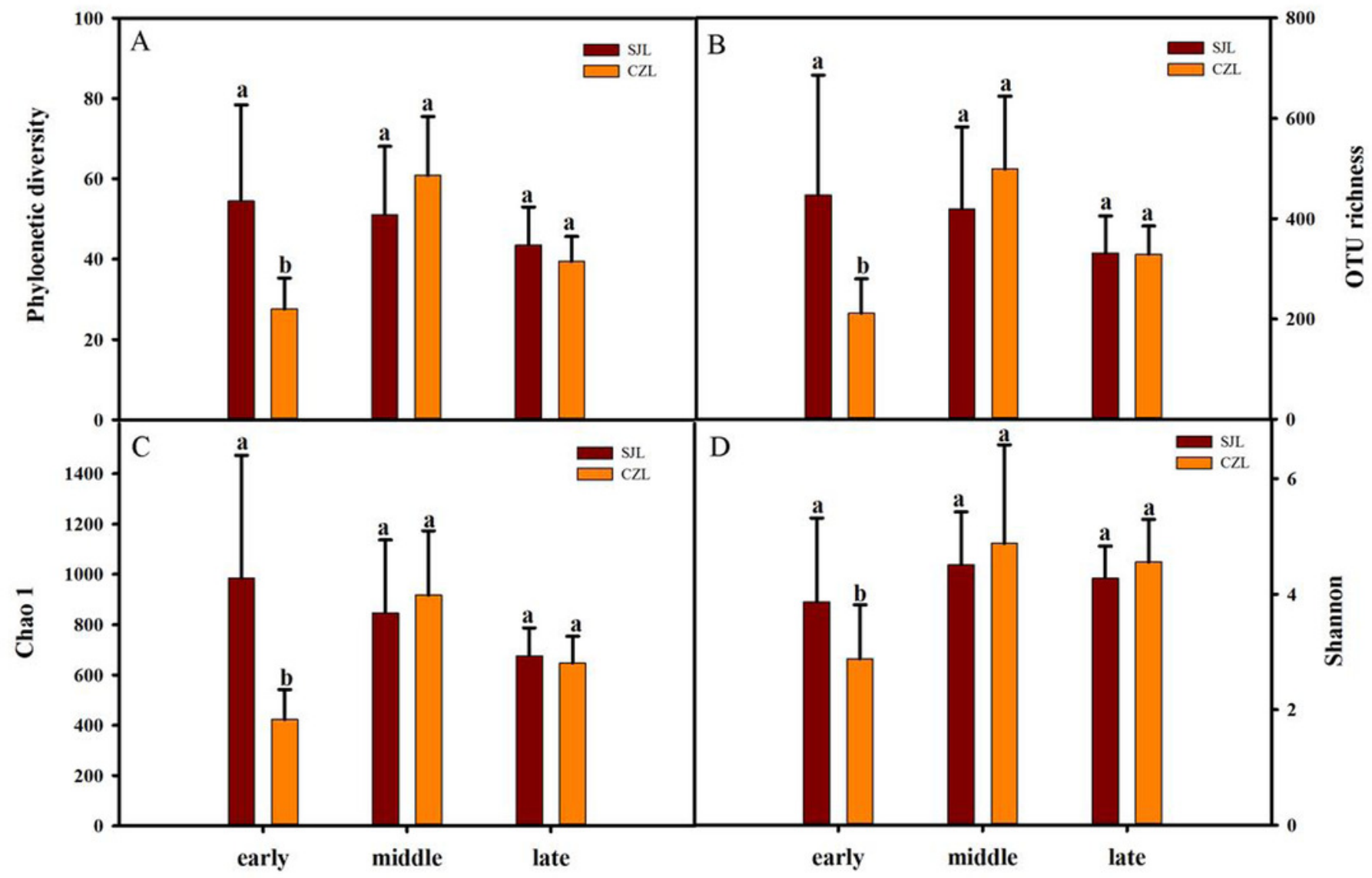




\section{Figure 3}

Relative abundance of gut bacteria.

Relative abundance of gut bacteria at the phylum, class and genus levels at two lakes. (A) Relative abundance of the dominant phyla in all samples. (B) Relative abundance of the dominant classes in all samples; Rings represent corresponding phylum (Firmicutes in blue and Proteobacteria in red) for each of the most frequently represented class. (C) Relative abundance at the phylum and (D) relative abundance at the genera at different periods in the two lakes.
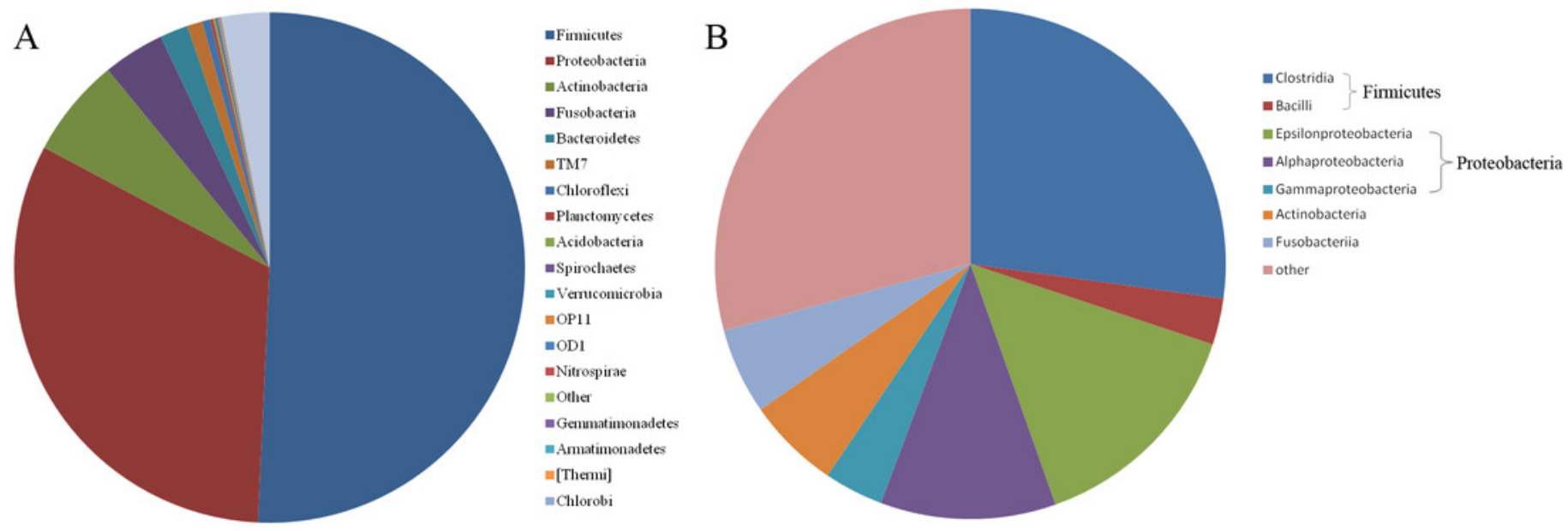

$\mathrm{C}$

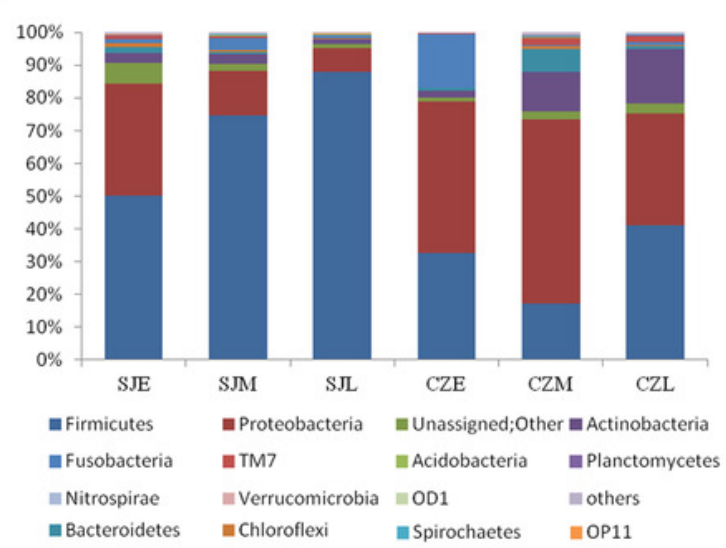

D

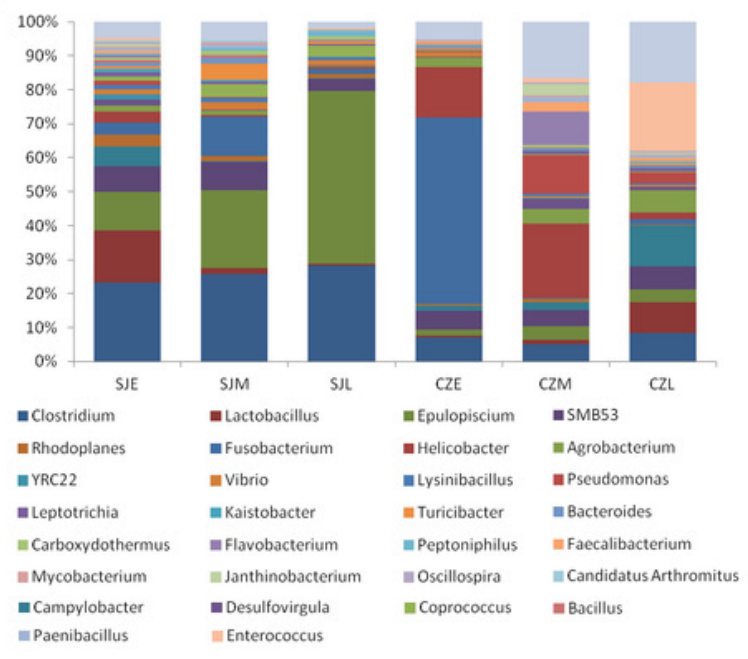


Figure 4

Relative abundance of the dominant phyla.

Relative abundance of the dominant phyla ( (A) Firmicutes, (B) Ptoteobacteria, (C)

Actinobacteria, (D) Fusobacteria) in different wintering periods. Samples are grouped

according to sampling location and wintering periods. Different letters represent significant differences by Tukey's HSD comparisons $(P<0.05)$. Error bars indicate standard deviation.

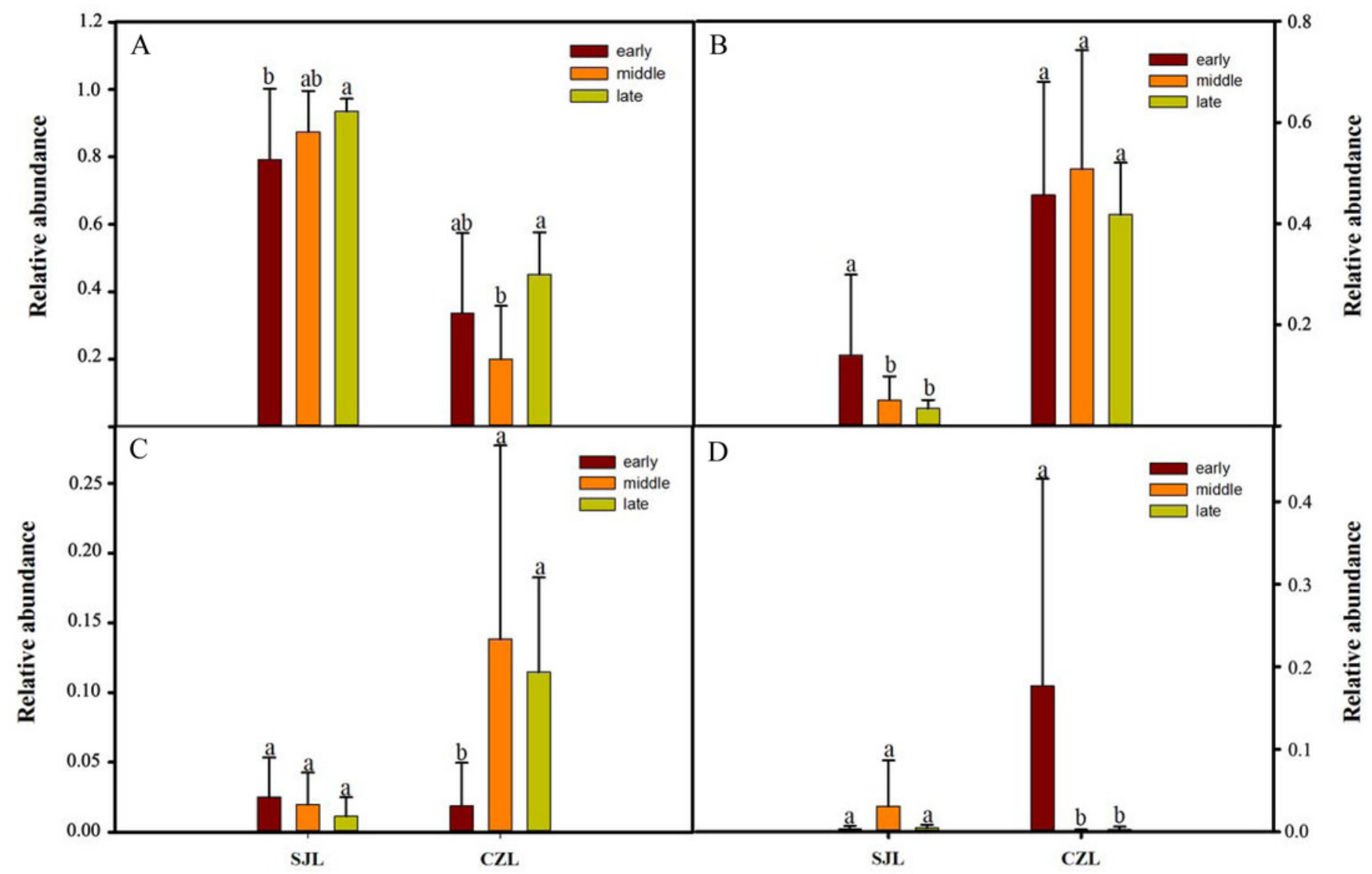




\section{Figure 5}

LEFSe analysis of the Hooded Crane guts bacteria.

LEFSe analysis of the Hooded Crane guts bacteria in different wintering periods (LDA $>2, P<$ 0.05). (A) LDA score representing the taxonomic with significant difference in different wintering periods at the Shengjin Lake. (B) Cladogram representing the taxonomic hierarchical structure of the phylotype biomakera identified among three wintering periods from the Shengjin Lake; Red, phylotypes statistically overrepresented in early wintering period; Green, phylotypes statistically overrepresented in late wintering period. (C) LDA score representing the taxonomic with significant difference in different wintering periods at the Caizi Lake. (D) Cladogram represting the taxonomic hierarchical structure of the phylotype biomakera identified among three wintering periods from the Caizi Lake; Red, phylotypes statistically overrepresented in early wintering period; Green, phylotypes statistically overrepresented in late wintering period; Blue, phylotypes statistically overrepresented in middle wintering period. 


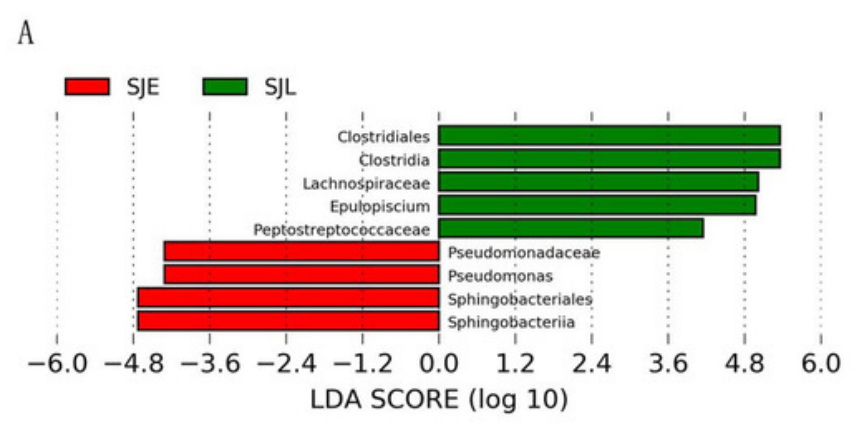

B $\square$ SJE

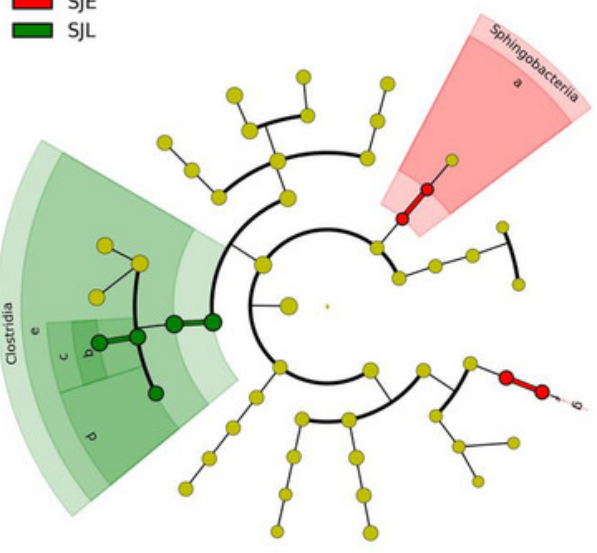

a: Sphingobacteriales

b: Epulopiscium

d: Peptostreptococcaceae e: Clostridiales

g: Pseudomonadaceae

C

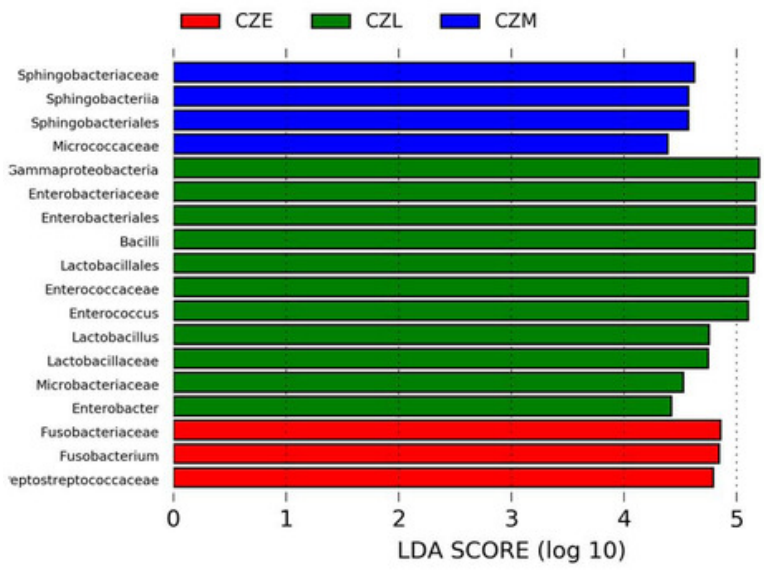

D

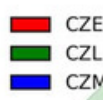

CZM

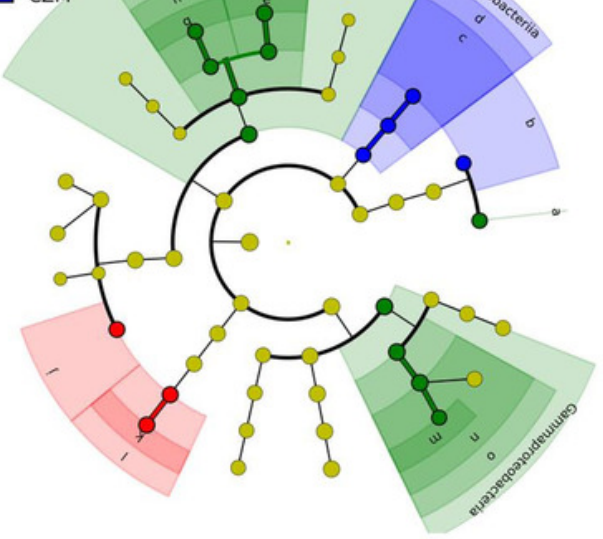

a: Microbacteriaceae - sphingobaceserince d: Sphingobacteriales e: Enterococcus f: Enterococcacea g: Lactobacillus hi Lactobacillaceae i: Lactobacillales i: Peptostreptococcacea k: Fusobacterium m: Enterobacter n: Enterobacteriace o: Enterobacteriales 


\section{Figure 6}

Differences in gut bacterial community composition.

Differences in gut bacterial community composition. (A) Non-metric multidimensional scaling plot bacterial community composition from Shengjin and Caizi Lakes at three wintering periods. (B) Non-metric multidimensional scaling plot showing bacterial community composition from Shengjin and Caizi Lakes at the early wintering period. (C) Non-metric multidimensional scaling plot for the hooded crane from Shengjin and Caizi Lakes at the middle wintering period. (D) Non-metric multidimensional scaling plot for the hooded crane from Shengjin and Caizi Lakes at the late wintering period. (E) Non-metric multidimensional scaling plot for the hooded crane from Shengjin Lake at three wintering periods. (F) Nonmetric multidimensional scaling plot for the hooded crane from Caizi Lake at three wintering periods. 


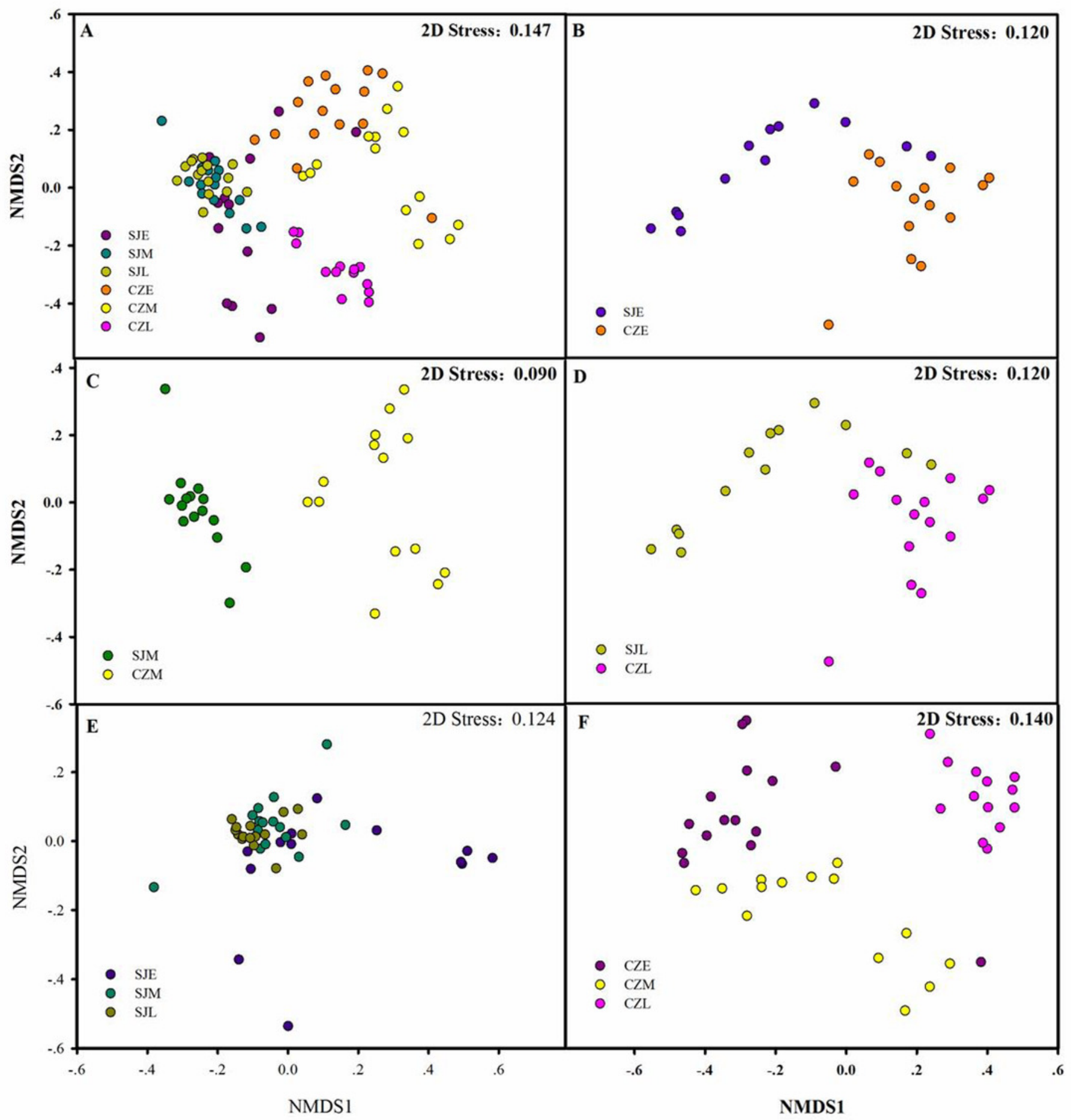


Figure 7

Bacterial phylogenetic structure evaluated by the NTI

Bacterial phylogenetic structure evaluated by the NTI in different wintering periods. (A) At the early wintering period, (B) At the middle wintering period, (C)At the late wintering period.

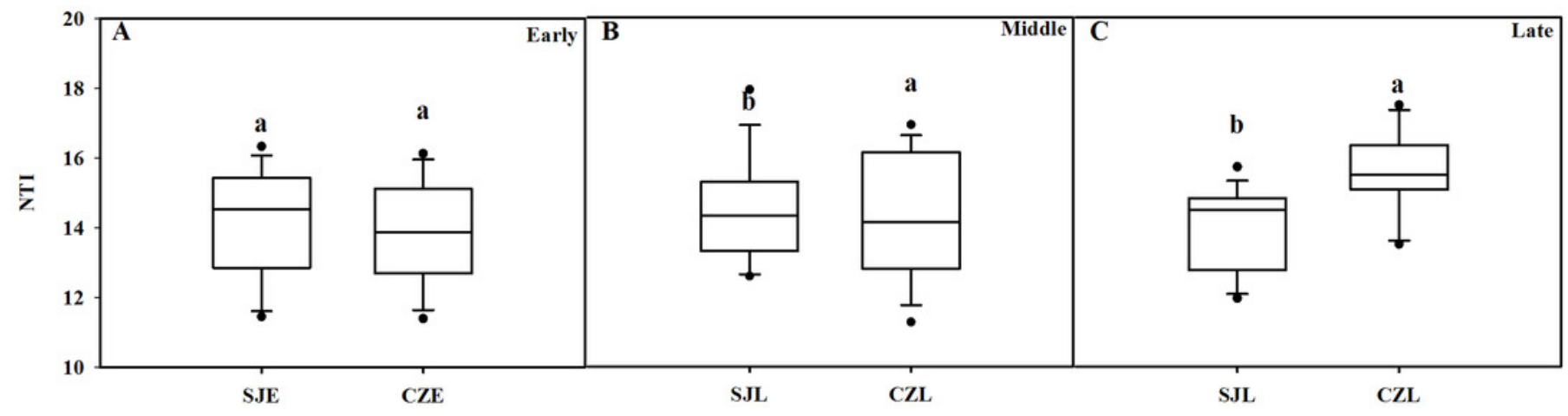




\section{Table $\mathbf{1}$ (on next page)}

ANOSIM analysis

Differences in the microbial community composition based on the similarity test of ANOSIM. 
1 Table1. Differences in the microbial community composition based on the similarity test of 2 ANOSIM.

\begin{tabular}{|c|c|c|c|c|c|}
\hline & \multicolumn{2}{|c|}{ temporal variation } & & \multicolumn{2}{|c|}{ spatial variation } \\
\hline & $\mathrm{R}$ & $P$ & & $\mathrm{R}$ & $P$ \\
\hline SJE vs SJM & 0.264 & 0.001 & SJE vs CZE & 0.591 & 0.001 \\
\hline SJM vs SJL & 0.124 & 0.009 & SJM vs CZM & 0.85 & 0.001 \\
\hline CZE vs CZM & 0.4 & 0.001 & SJL vs CZL & 0.967 & 0.001 \\
\hline CZM vs CZL & 0.845 & 0.001 & & & \\
\hline SJE vs SJM vs SJL & 0.238 & 0.001 & & & \\
\hline CZE vs CZM vs CZL & 0.739 & 0.001 & & & \\
\hline
\end{tabular}

3 
Table 2 (on next page)

ANOSIM analysis

Differences in the microbial functions based on the similarity test of ANOSIM. 
1 Table 2. Differences in the microbial functions based on the similarity test of ANOSIM. 2

\begin{tabular}{|c|c|c|c|c|c|}
\hline & \multicolumn{2}{|c|}{ temporal variation } & & \multicolumn{2}{|c|}{ spatial variation } \\
\hline & $\mathrm{R}$ & $\mathrm{P}$ & & $\mathrm{R}$ & $\mathrm{P}$ \\
\hline SJE vs SJM vs SJL & 0.181 & 0.005 & SJE vs CZE & 0.444 & 0.001 \\
\hline \multirow[t]{2}{*}{ CZE vs CZM vs CZL } & 0.218 & 0.001 & SJM vs CZM & 0.058 & 0.116 \\
\hline & & & SJL vs CZL & 0.221 & 0.001 \\
\hline
\end{tabular}

3 